\title{
Autosomal Dominant Alport Syndrome Presenting as Proteinuria at Marine Corps Physical Fitness Test: A Case Report and Review
}

Wisit Cheungpasitporn ${ }^{1 *}$, Quanhathai Kaewpoowat ${ }^{1}$, Promporn Suksaranjit' ${ }^{1}$, Wonngarm Kittanamongkolchai ${ }^{1}$, Narat Srivali ${ }^{1}$, Patompong Ungprasert ${ }^{1}$ and Yashaswini Rangan ${ }^{2}$

${ }^{1}$ Department of Internal Medicine, Bassett Medical Center, Cooperstown, New York 13326, USA

${ }^{2}$ Department of Internal Medicine, Division of Nephrology, Bassett Medical Center and Columbia University College of Physicians and Surgeons, Cooperstown, New York 13326, USA

\begin{abstract}
A 19-year-old Caucasian male presented to Nephrology Clinic for evaluation of proteinuria. He denied any hearing or vision impairment. The patient reported significant family history for kidney problem in his father, paternal uncle, paternal aunts and his half brother who shared the same father. Physical examination revealed a blood pressure of $136 / 60 \mathrm{mmHg}$ with no peripheral edema. Laboratory evaluation disclosed a serum creatinine of $0.7 \mathrm{mg} / \mathrm{dl}$, 24-hour urine protein of $2.4 \mathrm{~g}$ and serum albumin of $3.7 \mathrm{~g} / \mathrm{dl}$. Urinalysis demonstrated dysmorphic red blood cells. A renal biopsy revealed the diagnosis of Alport syndrome. From his paternal family history, the disease was transmitted by autosomal dominant inheritance. The diagnosis of autosomal dominant Alport syndrome was made. He was prescribed lisinopril $5 \mathrm{mg}$ per day. Referrals with Ophthalmology and Audiology were performed with showed no evidences of extrarenal involvement. At follow-up, 3 months later, patient continued to do well with serum creatinine of $0.7 \mathrm{mg} / \mathrm{dl}$ and urine protein-to-creatinine ratio of 1.75 .
\end{abstract}

Alport syndrome is most commonly transmitted in an X-linked manner ( $80 \%$ of cases). Autosomal dominant transmission is rare and only accounts for $5 \%$ of affected patients. Extrarenal manifestations include hearing loss and ocular defects. Although the presence of these defects should prompt a search for Alport syndrome, patients with autosomal dominant Alport syndrome may present without these manifestations.

Keywords: Autosomal dominant; Alport syndrome; Hematuria; Hereditary nephritis; Proteinuria

Abbreviation: ACEI: Angiotensin-Converting Enzyme Inhibitors; ANA: Anti Nuclear Antibody; ARB: Angiotensin II Receptor Blockers; C3: Complement 3; C4: Complement 4; ESRD: End Stage Renal Disease; GBM: Glomerular Basement Membrane

\section{Introduction}

Alport syndrome or hereditary nephritis is a rare genetic disorder of the glomerulus with an incidence of 1 in 50,000 live births [1]. The disease is caused by abnormalities in type IV collagen, a key component of the glomerular basement membrane (GBM). Hematuria is the initial renal presentation of the disease, and often presents in childhood. Common extrarenal manifestations include sensorineural hearing loss and lenticonus of the anterior lens capsule. Classic Alport syndrome is inherited in an X-linked pattern and is the most common form of Alport syndrome ( $80 \%$ of cases). Autosomal recessive variant accounts for approximately $15 \%$ of cases. Autosomal dominant transmission is rare and only accounts for $5 \%$ of affected patients [1]. We report a case of autosomal dominant Alport syndrome presenting as asymptomatic proteinuria without significant extrarenal manifestations.

\section{Case Presentation}

A 19-year-old Caucasian-American male presented to Nephrology Clinic for evaluation of proteinuria. He was in the process of undergoing a physical for the Marine Corps and was found to have proteinuria. His past medical history was significant for asymptomatic microscopic hematuria and bilateral hip pain from femoro-acetabular impingement syndrome for which he occasionally took ibuprofen. $\mathrm{He}$ denied any hearing or vision impairment.

The patient reported significant family history of kidney disease (Figure 1) in his father, paternal uncle and paternal aunts. His half brother who shared the same father also had "red blood cells in his urine". However, none of them had needed hemodialysis or kidney transplantation yet. Physical examination revealed a blood pressure of $136 / 60 \mathrm{mmHg}$ with no peripheral edema. There was no evidence of hearing loss, lenticonus of the anterior lens capsule, or abnormal lumps on the skin.

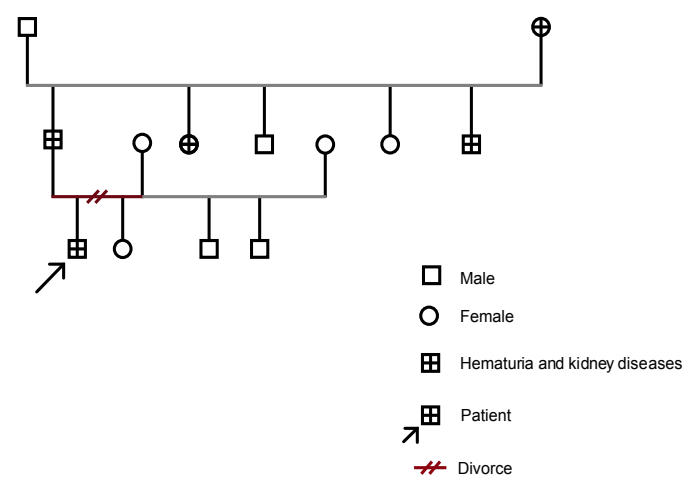

Figure 1: The patient's family genogram.

*Corresponding author: Wisit Cheungpasitporn, Department of Internal Medicine Bassett Medical Center, Cooperstown, New York 13326, USA, E-mail: Wisit. Cheungpasitporn@bassett.org

Received March 13, 2012; Accepted April 28, 2012; Published April 30, 2012

Citation: Cheungpasitporn W, Kaewpoowat Q, Suksaranjit P, Kittanamongkolcha W, Srivali N, et al. (2012) Autosomal Dominant Alport Syndrome Presenting as Proteinuria at Marine Corps Physical Fitness Test: A Case Report and Review. Nephrol Therapeut S8:001. doi:10.4172/2161-0959.S8-001

Copyright: (c) 2012 Cheungpasitporn W, et al. This is an open-access article distributed under the terms of the Creative Commons Attribution License, which permits unrestricted use, distribution, and reproduction in any medium, provided the original author and source are credited. 
Citation: Cheungpasitporn W, Kaewpoowat Q, Suksaranjit P, Kittanamongkolchai W, Srivali N, et al. (2012) Autosomal Dominant Alport Syndrome Presenting as Proteinuria at Marine Corps Physical Fitness Test: A Case Report and Review. J Nephrol Therapeut S8:001. doi:10.4172/2161 0959.S8-001

Page 2 of 3

Laboratory testing showed no anemia (HGB $16.1 \mathrm{gm} / \mathrm{dl}$, HCT 44.8 $\%$, MCV $85.0 \mathrm{fl}$, and MCH $30.6 \mathrm{pg}$ ), serum creatinine of $0.7 \mathrm{mg} / \mathrm{dl}, 24-$ hour urine protein of $2.4 \mathrm{~g}$, serum albumin of $3.7 \mathrm{~g} / \mathrm{dl}$ and $\mathrm{HbA1C}$ of 4.7 $\%$. Microscopic urianalysis demonstrated dysmorphic red blood cells (75-100/HPF). No abnormalities were noted on kidney ultrasound. Serologic workup was negative for anti-nuclear antibody, hepatitis B surface antigen, hepatitis $\mathrm{C}$ antibody and HIV.

Based on the family history, the pattern of inheritance was consistent with autosomal dominant. The patient subsequently underwent a kidney biopsy. Microscopic examination showed intact cortical tubules and normocellular glomeruli (Figure 2). Immunofluorescence revealed no significant staining. Electron microscopy revealed segmental to global GBM lamellation and segmental GBM thinning (Figure 3), diagnostic of Alport syndrome.

The patient was prescribed lisinopril $5 \mathrm{mg}$ per day to reduce proteinuria. Referrals to Ophthalmology and Audiology were performed with showed no evidence of extrarenal involvement. Three months later, patient's serum creatinine was $0.7 \mathrm{mg} / \mathrm{dL}$ and urine protein-to-creatinine ratio was 1.75

\section{Discussion}

Alport syndrome, first identified by Dr. Cecil A. Alport in 1927 [2], is due to abnormalities of type IV collagen, a key component of the GBM. Type IV collagen molecules are composed of three alpha chains that form triple-helical structures through specific interactions of $\mathrm{C}$-terminal noncollagenous domains and are made up of heterotrimers composed of combinations of 6 possible alpha chains [3]. These 6 distinct type IV collagen chains are encoded by 6 different genes that are arranged in pairs on 3 different chromosomes: COL4A1 and COL4A2 are on chromosome 13; COL4A3 and COL4A4 are on chromosome 2; and COL4A5 and COL4A6 are on chromosome X. The mutation of COL4A5 gene causes the classic X-linked form of Alport syndrome (85\% of cases). Autosomal recessive inheritance arises from genetic defects in either COL4A3 or COL4A4 genes. Rare cases of autosomal

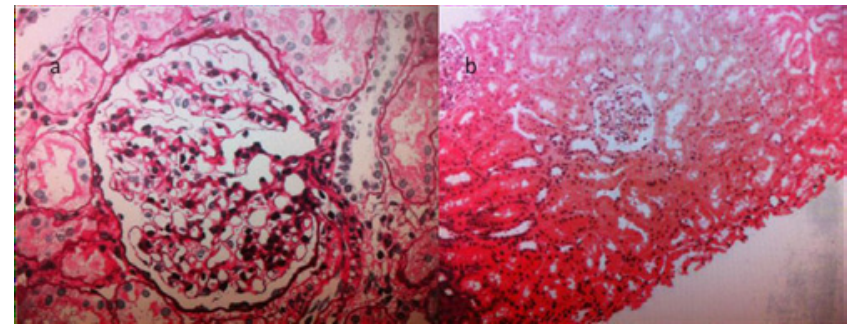

Figure 2: Microscopic examination revealed normocellular glomerulus (a) and intact cortical tubules (b)

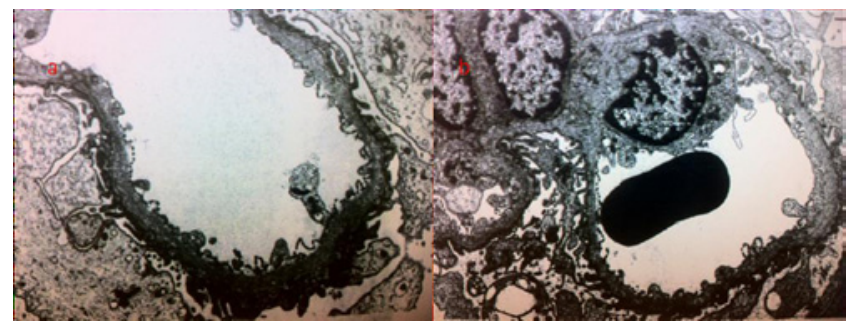

Figure 3: Electron microscopy $(x 10,000)$ demonstrated segmental to globa GBM lamellation and segmental GBM thinning, diagnostic of hereditary nephritis. dominant Alport syndrome due to heterozygous mutation in COL4A3 or COL4A4 also occur [1-4].

Clinical features of Alport syndrome including hematuria, sensorineural hearing loss, ocular defects and progression to renal failure are often seen in early adulthood. Hematuria is the most common (all males and $95 \%$ of affected female) and earliest manifestation, although some proteinuria may also present as in our case vignette. End Stage Renal Disease (ESRD) usually occurs between the ages of 16 and 35 years in patients with X-linked or autosomal recessive disease. The clinical features of autosomal dominant Alport syndrome are similar to those of X-linked disease. However, deterioration of renal function tends to occur more slowly [5-7].

Bilateral sensorineural hearing loss typically does not occur until adulthood. In general, the rate of hearing loss is similar to the progression of renal insufficiency. Ocular defects occur in up to a third of patients. Anterior lenticonus is the most common eye defect and pathognomonic of the disease. However, patients with the autosomal dominant form usually have less ocular abnormalities [8]. Leiomyomas are found in 2 to 5 percent of patients and carriers of X-linked Alport syndrome and are typically not seen in autosomal patterns [9] The patient in our case study presented with non-nephrotic range proteinuria and microscopic hematuria without any evidence of ocular or hearing defects, and renal insufficiency.

In a kidney biopsy specimen, light microscopy may show no significant abnormalities in early stages. Glomerulosclerosis, interstitial fibrosis and prominent foam cells are seen in later stages. Immunofluorescence may show non-trapping of IgM. The characteristic finding of longitudinal splitting of the lamina densa of the GBM detected by electron microscopy (Figure 2) is diagnostic of Alport syndrome. A less invasive potential method to diagnose a child with suspected X-linked Alport syndrome is a skin biopsy using monoclonal antibody against the alpha-5 type IV collagen chain; however, this test cannot detect autosomal inheritance forms which are caused by mutation in COL4A3 and COL4A4 genes [10].

Differential diagnosis of Alport syndrome includes other glomerular disorders that present in children with microscopic hematuria such as IgA nephropathy and thin basement membrane nephropathy. In IgA nephropathy, family history is usually negative. On the other hand, the family history may be positive for hematuria in thin basement membrane nephropathy, but renal failure and deafness are typically absent [11].

There is currently no specific treatment for Alport syndrome, although the use of angiotensin-converting enzyme inhibitors (ACEI), Angiotensin II Receptor Blockers (ARB) or cyclosporine [12] has been described in uncontrolled studies. A number of reports showed evidence of decreased proteinuria in response to ACEI [13-16]. In patients who develop ESRD, the preferred modality of renal replacement therapy is transplantation. Although approximately $3 \%$ of transplanted males develop de novo anti-GBM disease [17], there is currently no report of recurrent Alport syndrome in the transplanted individual. Although the patient in our case report was normotensive, he was prescribed an ACEI to reduce proteinuria. At follow-up, the patient continued to do well without progression of disease.

\section{Conclusion}

The clinical manifestations of autosomal dominant Alport syndrome are similar to those of X-linked inheritance. However, deterioration of kidney function tends to occur more slowly. Visual 
Citation: Cheungpasitporn W, Kaewpoowat Q, Suksaranjit P, Kittanamongkolchai W, Srivali N, et al. (2012) Autosomal Dominant Alport Syndrome Presenting as Proteinuria at Marine Corps Physical Fitness Test: A Case Report and Review. J Nephrol Therapeut S8:001. doi:10.4172/2161 0959.S8-001

Page 3 of 3

and hearing defects are characteristic features observed in patients with Alport syndrome, but do not occur in those with thin basement membrane disease, a relatively more common cause of asymtomatic hematuria. Although the presence of ocular or hearing defects should prompt a search for Alport syndrome, patients with autosomal dominant Alport syndrome can present without these manifestations and may hence prove harder to diagnose.

\section{Consent}

The patient provided written consent for the publication of this case report and any accompanying images. A copy of the written consent is available for review by the journal's Editor-in-Chief.

\section{Acknowledgements}

We acknowledge Dr. Edward F Bischof, Program Director of the Internal Medicine Residency Program at Bassett Medical Center, who always encourages us to study our patients' cases.

\section{References}

1. Pescucci C, Mari F, Longo I, Vogiatzi P, Caselli R, et al. (2004) Autosomaldominant Alport syndrome: Natural history of a disease due to COL4A3 or COL4A4 gene. Kidney Int 65:1598-1603.

2. Alport AC (1927) Hereditary familial congenital haemorrhagic nephritis. Br Med J 1: 504-506.

3. Hudson BG (2004) The molecular basis of Goodpasture and Alport syndromes: beacons for the discovery of the collagen IV family. J Am Soc Nephrol 15: 2514-2527.

4. Longo I, Porcedda P, Mari F, Giachino D, Meloni I, et al. (2002) COL4A3/ COL4A4 mutations: From familial hematuria to autosomal-dominant or recessive Alport syndrome. Kidney Int 61: 1947-1956.

5. Pochet JM, Bobrie G, Landais P, Goldfarb B, Grunfeld JP, et al. (1989) Renal prognosis in Alport's and related syndromes: influence of the mode of inheritance. Nephrol Dial Transplant 4:1016-1021.
6. Marcocci E, Uliana V, Bruttini M, Artuso R, Silengo MC, et al. (2009) Autosomal dominant Alport syndrome: molecular analysis of the COL4A4 gene and clinical outcome. Nephrol Dial Transplant 24: 1464-1471.

7. Kharrat M, Makni S, Makni K, Kammoun K, Charfeddine K, et al. (2006) Autosomal dominant Alport's syndrome: study of a large Tunisian family. Saud J Kidney Dis Transpl 17: 320-325.

8. Grünfeld JP (1985) The clinical spectrum of hereditary nephritis. Kidney Int 27: 83-92.

9. Dahan K, Heidet L, Zhou J, Mettler G, Leppig KA, et al. (1995) Smooth muscle tumors associated with $\mathrm{X}$-linked Alport syndrome: carrier detection in females. Kidney Int 48: 1900-1906.

10. van der Loop FT, Monnens LA, Schröder $\mathrm{CH}$, Lemmink $\mathrm{HH}$, Breuning $\mathrm{MH}$ et al. (1999) Identification of COL4A5 defects in Alport's syndrome by immunohistochemistry of skin. Kidney Int 55: 1217-1224.

11. TieboschAT,FrederikPM, vanBredaVriesmanPJ,MooyJM, vanReiH, etal.(1989) Thin-basement-membrane nephropathy in adults with persistent hematuria. $\mathrm{N}$ Eng J Med 320: 14-18.

12. Callís L, Vila A, Carrera M, Nieto J (1999) Long-term effects of cyclosporine A in Alport's syndrome. Kidney Int 55: 1051-1056.

13. 13)Webb NJ, Lam C, Shahinfar S, Strehlau J, Wells TG, et al. (2011) Efficacy and safety of losartan in children with Alport syndrome--results from a subgroup analysis of a prospective, randomized, placebo- or amlodipine-controlled trial. Nephrol Dial Transplant 26: 2521-2526.

14. Cohen EP, Lemann J Jr. (1996) In hereditary nephritis angiotensin-converting enzyme inhibition decreases proteinuria and may slow the rate of progression. Am J Kidney Dis 27: 199-203.

15. Proesmans W, Knockaert H, Trouet D (2000) Enalapril in paediatric patients with Alport syndrome: 2 years' experience. Eur J Pediatr 159: 430-433.

16. Proesmans W, Van Dyck M (2004) Enalapril in children with Alport syndrome. Pediatr Nephrol 19: 271-275

17. Kashtan CE, Michael AF (1966) Alport syndrome. Kidney Int 50: 1445-1463.
This article was originally published in a special issue, Clinical Glomerulonephiritis handled by Editor(s). Dr. Ahmed Adel Hassan, Zagazig University, Egypt 